\title{
Integration of the Tomonaga-Schwinger Equation
}

\author{
Hitoshi Wakita \\ Department of Applied Mathematics, Faculty of Engineering, Gifu University, \\ Kagamihara, Japan
}

\begin{abstract}
Some integrations of the Tomonaga-Schwinger equation with a non-local interaction are studied with mathematical rigor. It is proved that the related initial value problem has a unique solution in any finite region of the space-time corresponding to each set of space-like surfaces which covers the region. Such an analysis can be extended to the case of quantum electrodynamics by the aid of a Lorentz-invariant topology introduced in the *-algebra of electromagnetic field operators.
\end{abstract}

\section{§ 1. Introduction}

Though the Tomonaga-Schwinger equation

$$
i \delta \Psi(\sigma) / \delta \sigma(x)=H(x) \Psi(\sigma)
$$

in the interaction picture is most fundamental in the early quantum field theory, it is very difficult to understand the precise mathematical meaning of this equation. If, however, we replace the interaction Hamiltonian density $H(x)$ by some nonlocal ones, we get a clue to treat the equation mathematically. The purpose of the present paper is to study this problem in some detail.

We start with the scalar coupling between a neutral scalar field of mass $\mu \neq 0$ and a spinor field of mass $\kappa \neq 0$. We work with the Hilbert space

$$
\mathfrak{H}=\mathfrak{H}_{M} \otimes \mathfrak{H}_{D},
$$

where $\mathfrak{G}_{M}$ is the Fock space for creation and annihilation operator-valued distributions $\phi^{*}(\boldsymbol{k})$ and $\phi(\boldsymbol{k})$ of the neutral scalar field and $\mathfrak{S}_{D}$ is the same for the spinor field. We are interested in the local interaction Hamiltonian density

$$
H(x)=g N[\tilde{\psi}(x) \psi(x)] \phi(x)^{1},
$$

$1 \quad N[\ldots]$ means the normal product 
where $\phi(x)$ is the neutral scalar field

$$
\left.\begin{array}{rl}
\phi(x) & =\phi^{+}(x)+\phi^{-}(x), \\
\phi^{+}(x) & =2^{-1 / 2}(2 \pi)^{-3 / 2} \int \exp (+i k x) \phi(\boldsymbol{k}) d \boldsymbol{k} / k^{0}, \\
\phi^{-}(x) & =2^{-1 / 2}(2 \pi)^{-3 / 2} \int \exp (-i k x) \phi^{*}(\boldsymbol{k}) d \boldsymbol{k} / k^{0},
\end{array}\right\}
$$

and $\psi(x)$ is the spinor field, both in the interaction picture. Instead of attacking (1.2), however, we shall consider the regularized Hamiltonian density

$$
H(x ; \varrho)=g N\left[\tilde{\psi}\left(x ; \varrho_{D}\right) \psi\left(x ; \varrho_{D}\right)\right] \phi\left(x ; \varrho_{M}\right),
$$

where

$$
\left.\begin{array}{l}
\phi(x ; \varrho)=\int \varrho_{M}(x-y) \phi(y) d y, \\
\psi(x ; \varrho)=\int \varrho_{D}(x-y) \psi(y) d y, \\
\tilde{\psi}(x ; \varrho)=\int \varrho_{D}(x-y) \tilde{\psi}(y) d y,
\end{array}\right\}
$$

and regulators $\varrho_{M}(x)$ and $\varrho_{D}(x)$ are to satisfy conditions described in the next section.

The derivative on the left-hand side of (1.1) is defined in the following manner. We introduce a covering $\left\{\sigma_{\lambda}\right\}$ of a space-time region in terms of one-parameter family of space-like surfaces

$$
\sigma_{\lambda}=\left\{\left(x^{0}(x ; \lambda), x\right)\right\},
$$

which are to satisfy conditions given in the next section. We always put the restriction that the region $\Sigma\left(\sigma_{b} \succ \sigma_{a}\right)$ between two space-like surfaces $\sigma_{b}$ and $\sigma_{a}$ is bounded. The derivative in question is then defined by

$$
\frac{\delta \Psi\left(\sigma_{\lambda}\right)}{\delta \sigma_{\lambda}}=\lim _{\Delta \lambda \rightarrow+0} \frac{\Psi\left(\sigma_{\lambda+\Delta \lambda}\right)-\Psi\left(\sigma_{\lambda}\right)}{\left|\Sigma\left(\sigma_{\lambda+\Delta \lambda} \succ \sigma_{\lambda}\right)\right|}
$$

where the denominator is the volume. Because of the formula

$$
d\left|\Sigma\left(\sigma_{\lambda}>\sigma_{a}\right)\right| / d \lambda=\int\left(\partial x^{0} / \partial \lambda\right) d x,
$$

we can interprete the formula (1.1) as

$$
i d \Psi\left(\sigma_{\lambda}\right) / d \lambda=H(\lambda ; \varrho) \Psi\left(\sigma_{\lambda}\right),
$$

where

$$
H(\lambda ; \varrho)=\int H(x ; \varrho)\left(\partial x^{0} / \partial \lambda\right) d x .
$$

The main purpose of this paper is to point out that the Equation $\left(1.1^{\prime}\right)$ has a unique solution $U\left(\sigma_{\lambda}, \sigma_{\lambda_{0}}\right) \Psi\left(\sigma_{\lambda_{0}}\right)$ for any initial data $\Psi\left(\sigma_{\lambda_{0}}\right)$. The advantage of the present approach over the usual method of using only hyperplanes is obvious from (1.8). We have a spatial cut-off $\left(\partial x^{0} / \partial \lambda\right)$ without artificially introducing it. On the other hand, the assumption of bounded $\Sigma\left(\sigma_{a}>\sigma_{b}\right)$ does not seem to cause any inconvenience because any space-time point can be included inside a bounded $\Sigma\left(\sigma_{a} \succ \sigma_{b}\right)$.

The above analysis can be extended to quantum electrodynamics, as we shall see in Section 4 of this paper. The main difference from the preceding situation is 
that a Lorentz covariant field for electromagnetic potential can be introduced only on a (pseudo-)Hilbert space with indefinite metric. We overcome this difference by introducing a Lorentz-invariant topology on the *-algebra of field operators and taking into consideration continuous linear functionals which are not necessarily positive. We can then obtain a similar result as before, where $U\left(\sigma_{\lambda}, \sigma_{\lambda_{0}}\right)$ now describe a linear mapping of certain class of such continuous linear functionals.

\section{§ 2. Details of Technical Definitions}

In this section, we collect technical details in our paper. The regulator $\varrho(x)\left[\varrho_{M}(x)\right.$ or $\left.\varrho_{D}(x)\right]$ is of the form

$$
\varrho(x)=(2 \pi)^{-4} \int \exp (i k x) \varrho(k) d k,
$$

where $\varrho(k)$ satisfies the following:

$\left(\mathrm{R}_{1}\right) \hat{\varrho}(-k)=\bar{\varrho}(k)$; namely, $\varrho(x)$ is real.

$\left(\mathrm{R}_{2}\right) \quad \hat{\varrho}_{M}(\boldsymbol{k})=\left.\hat{\varrho}_{M}(k)\right|_{k^{0}=\left(\boldsymbol{k}^{2}+\mu^{2}\right)^{1 / 2} \in} \in(\boldsymbol{K})$, $\hat{\varrho}_{D}(\boldsymbol{k})=\left.\hat{\varrho}_{D}(k)\right|_{k^{0}=\left(\boldsymbol{k}^{2}+k^{2}\right)^{1 / 2} \in} \boldsymbol{\Xi}(\boldsymbol{K})$.

For two space-like surfaces $\sigma_{b}$ and $\sigma_{a}$, we write $\sigma_{b} \succ \sigma_{a}$ if $x^{0}(\boldsymbol{x} ; b) \geqq x^{0}(\boldsymbol{x} ; a)$ for all $\boldsymbol{x}$. The family $\left\{\sigma_{\lambda} ; b \geqq \lambda \geqq a\right\}$ of space-like surfaces [called covering of the region $\left.\Sigma\left(\sigma_{b} \succ \sigma_{a}\right)\right]$ is assumed to satisfy the following conditions:

$\left(\mathrm{C}_{1}\right)$ Any point $x \in \Sigma\left(\sigma_{b} \succ \sigma_{a}\right)$ belongs to at least one $\sigma_{\lambda}$.

$\left(C_{2}\right)$ The function $x^{0}(x ; \lambda)$ is differentiable with respect to $\lambda, x^{0}(x ; \lambda)$ and $\partial x^{0} / \partial \lambda$ are continuous with respect to $(\boldsymbol{x}, \lambda)$, and $\partial x^{0} / \partial \lambda \geqq 0$.

The condition $\left(\mathrm{C}_{2}\right)$ implies that $\sigma_{\lambda_{1}} \succ \sigma_{\lambda_{2}}$ if $\lambda_{1}, \lambda_{2} \in[a, b]$ and $\lambda_{1} \geqq \lambda_{2}$. It also implies that the volume $\left|\Sigma\left(\sigma_{\lambda} \succ \sigma_{a}\right)\right|$ is differentiable and (1.8) holds.

Let $\mathfrak{H}_{M, N}$ be the subspace of $\mathfrak{H}_{M}$ spanned by vectors with not more than $N$ particles and $P_{N}$ be the projection onto $\mathfrak{H}_{M, N}$. Let $\phi^{ \pm}(x ; \varrho)$ be defined by the same equation as (1.6) from $\phi^{ \pm}(x)$ and put

$$
\phi_{N}^{ \pm}(x ; \varrho)=P_{N} \phi^{ \pm}(x ; \varrho) P_{N} .
$$

Then, $\phi_{N}^{ \pm}(x ; \varrho)$ are bounded operators with domain $\mathfrak{H}_{M}$, and

$$
\left\|\phi_{N}^{ \pm}(x ; \varrho)\right\|<N^{1 / 2}\left\|\varrho_{M}\right\|,
$$

where

$$
\left\|\varrho_{M}\right\|^{2}=\int \overline{\varrho_{M}(\boldsymbol{k})} \hat{\varrho}_{M}(\boldsymbol{k}) d \boldsymbol{k} / k^{0} .
$$

Hence,

$$
\phi_{N}(x ; \varrho)=P_{N} \phi(x ; \varrho) P_{N}=\phi_{N}^{+}(x ; \varrho)+\phi_{N}^{-}(x ; \varrho)
$$

is a bounded self-adjoint operator in $\mathfrak{H}_{M}$. Furthermore, we can show that $\phi_{N}(x ; \varrho)$ is uniformly continuous with respect to $x$ in the uniform operator topology ( $u$-topology for short); namely, for any given $\varepsilon>0$, there is a positive number $\delta$ such that, if $|\Delta \boldsymbol{x}|+\left|\Delta x^{0}\right|<\delta$, then

$$
\left\|\phi_{N}(x+\Delta x ; \varrho)-\phi_{N}(x ; \varrho)\right\|<\varepsilon
$$


for any $x$. For $\Psi \in \mathfrak{S}_{M, N}$

$$
\phi_{N+1}(x ; \varrho) \Psi=\phi(x ; \varrho) \Psi .
$$

We restrict the domain of $\phi(x)$ to $\bigcup_{N} \mathfrak{S}_{M, N}$, where it is essentially self-adjoint.

We note that $\psi(x ; \varrho)$ and $\tilde{\psi}(x ; \varrho)$ are bounded and uniformly continuous with respect to $x$ in the $u$-topology.

The above consideration shows that $H(x ; \varrho)$ is an essentially self-adjoint operator whose domain is

$$
\bigcup_{N=0}^{\infty} \mathfrak{H}_{N}=\bigcup_{N=0}^{\infty} \mathfrak{H}_{M, N} \otimes \mathfrak{H}_{D} .
$$

Let $P_{N}$ be the projection upon $\mathfrak{G}_{N}$. Then,

$$
H_{N}(x ; \varrho)=P_{N} H(x ; \varrho) P_{N}
$$

is a bounded self-adjoint operator with domain $\mathfrak{H}$,

$$
\left\|H_{N}(x ; \varrho)\right\|<c N^{1 / 2}\left\|\varrho_{D}\right\|^{2}\left\|\varrho_{M}\right\|,
$$

and further, $H_{N}(x ; \varrho)$ is uniformly continuous with respect to $x$ in the $u$-topology. It follows from (2.2) that

$$
H_{N+1}(x ; \varrho) \Psi=H(x ; \varrho) \Psi
$$

for any $\Psi \in \mathfrak{H}_{N}$.

\section{§ 3. Integrations of the Equation}

The interaction Hamiltonian

$$
H(\lambda ; \varrho)=\int H(x ; \varrho)\left(\partial x^{0} / \partial \lambda\right) d x
$$

on a surface $\sigma_{\lambda}$ can now be defined rigorously as follows: As $H_{N}(x ; \varrho)$ is continuous with respect to $x$ in the $u$-topology, it is easy to show that $H_{N}(x ; \varrho)\left(\partial x^{0} / \partial \lambda\right)$ is Bochner integrable $[1, \mathrm{~V}]$ on $\sigma_{\lambda}$ with respect to the volume element $d \boldsymbol{x}$. Hence,

$$
H_{N}(\lambda ; \varrho)=\int H_{N}(x ; \varrho)\left(\partial x^{0} / \partial \lambda\right) d x \quad \text { (Bochner) }
$$

is well-defined, it is continuous with respect to $\lambda$ in the $u$-topology, and

$$
\begin{aligned}
\left\|H_{N}(\lambda ; \varrho)\right\| & \leqq \int\left\|H_{N}(x ; \varrho)\right\|\left|\partial x^{0} / \partial \lambda\right| d x \\
& <N^{1 / 2} C(\varrho ; \lambda) .
\end{aligned}
$$

If a covering $\left\{\sigma_{\lambda}\right\}$ is specified for the region $\Sigma\left(\sigma_{b}>\sigma_{a}\right)$, then $C(\varrho ; \lambda)$ can be replaced by one constant $C(\varrho)$ irrespective of $\lambda$. Put

$$
H(\lambda ; \varrho) \Psi=H_{N+1}(\lambda ; \varrho) \Psi
$$

for any $\Psi \in \mathfrak{S}_{N}$. Then it follows that $H(\lambda ; \varrho)$ is an essentially self-adjoint operator with domain $\bigcup \mathfrak{S}_{N}$, and we can understand the Equation (3.1) in the sense that

$$
H(\lambda ; \varrho) \Psi=\int[H(x ; \varrho) \Psi]\left(\partial x^{0} / \partial \lambda\right) d \boldsymbol{x} \quad \text { (Bochner) }
$$


for any $\Psi \in \mathfrak{S}_{N}$.

Now, we consider the equation

$$
i d \Psi\left(\sigma_{\lambda}\right) / d \lambda=H_{N}(\lambda ; \varrho) \Psi\left(\sigma_{\lambda}\right)
$$

for the Hamiltonian given by (3.2). Put

$$
\begin{aligned}
U_{N}\left(\sigma_{\lambda}, \sigma_{\lambda_{0}}\right)= & \sum_{n=0}^{\infty}(-i)^{n} \int_{\lambda_{0}}^{\lambda} d \lambda_{n} \int_{\lambda_{0}}^{\lambda_{n}} d \lambda_{n-1} \ldots \int_{\lambda_{0}}^{\lambda_{2}} d \lambda_{1} \\
& {\left[H_{N}\left(\lambda_{n} ; \varrho\right) H_{N}\left(\lambda_{n-1} ; \varrho\right) \ldots H_{N}\left(\lambda_{1} ; \varrho\right)\right] . }
\end{aligned}
$$

As $H_{N}(\lambda ; \varrho)$ is continuous in the $u$-topology, each term of the right-hand side is well-defined in the sense of the Bochner integral, and the norm of the $n$th term is less than $(n !)^{-1} N^{n / 2} C^{n}(\varrho)\left|\lambda-\lambda_{0}\right|^{n}$. This series converges in the $u$-topology and defines a unitary operator $U_{N}\left(\sigma_{\lambda}, \sigma_{\lambda_{0}}\right)$. It is continuous with respect to $\lambda$ in the $u$-topology, and

$$
U_{N}\left(\sigma_{\lambda}, \sigma_{\lambda_{1}}\right) U_{N}\left(\sigma_{\lambda_{1}}, \sigma_{\lambda_{0}}\right)=U_{N}\left(\sigma_{\lambda_{1}}, \sigma_{\lambda_{0}}\right)
$$

We can show that $U_{N}\left(\sigma_{\lambda}, \sigma_{\lambda_{0}}\right)$ is differentiable in the $u$-topology and that

$$
\text { id } U_{N}\left(\sigma_{\lambda}, \sigma_{\lambda_{0}}\right) / d \lambda=H_{N}(\lambda ; \varrho) U_{N}\left(\sigma_{\lambda}, \sigma_{\lambda_{0}}\right) \text {. }
$$

This implies that the initial value problem of the Equation (3.3) has a unique solution given by

$$
\Psi\left(\sigma_{\lambda}\right)=U_{N}\left(\sigma_{\lambda}, \sigma_{\lambda_{0}}\right) \Psi\left(\sigma_{\lambda_{0}}\right)
$$

for any $\Psi\left(\sigma_{\lambda_{0}}\right) \in \mathfrak{H}$. As these assertions are very plausible, we omit the proofs ${ }^{2}$.

We are thus in a position to study the main problem of this article; namely, the initial value problem of the equation

$$
i d \Psi\left(\sigma_{\lambda}\right) / d \lambda=\tilde{H}(\lambda ; \varrho) \Psi\left(\sigma_{\lambda}\right)
$$

for the Hamiltonian $\tilde{H}(\lambda ; \varrho)$, the self-adjoint extension of $H(\lambda ; \varrho)$ given by $(3.1)$. Put

$$
\begin{aligned}
U\left(\sigma_{\lambda}, \sigma_{\lambda_{0}}\right) \Psi= & \sum_{n=0}^{\infty}(-i)^{n} \int_{\lambda_{0}}^{\lambda} d \lambda_{n} \int_{\lambda_{0}}^{\lambda_{n}} d \lambda_{n-1} \ldots \int_{\lambda_{0}}^{\lambda_{2}} d \lambda_{1} \\
& {\left[H\left(\lambda_{n} ; \varrho\right) H\left(\lambda_{n-1} ; \varrho\right) \ldots H\left(\lambda_{1} ; \varrho\right) \Psi\right] }
\end{aligned}
$$

for any $\Psi \in \bigcup \mathfrak{H}_{N}$. If $\Psi \in \mathfrak{H}_{N}$, the integrand of each term of the right-hand side is strongly continuous, and the Bochner integrals are well-defined. The norm of the $n$th term is less than

$$
(n !)^{-1}[(N+n) ! / N !]^{1 / 2} C^{n}(\varrho)\left|\lambda-\lambda_{0}\right|^{n}\|\Psi\|,
$$

and hence the series converges in the strong sense. This means that $U\left(\sigma_{\lambda}, \sigma_{\lambda_{0}}\right)$ is well-defined as an operator with domain $\bigcup \mathfrak{H}_{N}$. It is easy to show that

$$
U\left(\sigma_{\lambda}, \sigma_{\lambda_{0}}\right) \Psi=\lim _{N \rightarrow \infty} U_{N}\left(\sigma_{\lambda}, \sigma_{\lambda_{0}}\right) \Psi
$$

2 Some of related problems are studied in [1, XIV] and [2] 
for any $\Psi \in \bigcup \mathfrak{H}_{N}$ in the strong sense, and this implies that $U\left(\sigma_{\lambda}, \sigma_{\lambda_{0}}\right)$ can be extended as an isometric operator with domain $\mathfrak{H}$. This extended operator satisfies

$$
U\left(\sigma_{\lambda}, \sigma_{\lambda_{0}}\right)=\lim _{N \rightarrow \infty} U_{N}\left(\sigma_{\lambda}, \sigma_{\lambda_{0}}\right)
$$

in the strong operator topology, and from (3.5)

$$
U\left(\sigma_{\lambda}, \sigma_{\lambda_{1}}\right) U\left(\sigma_{\lambda_{1}}, \sigma_{\lambda_{0}}\right)=U\left(\sigma_{\lambda_{1}}, \sigma_{\lambda_{0}}\right) .
$$

In the same way, we can show by a direct calculation that, for any $\Psi \in \bigcup \mathfrak{H}_{N}$,

$$
\Psi\left(\sigma_{\lambda}\right)=U\left(\sigma_{\lambda}, \sigma_{\lambda_{0}}\right) \Psi
$$

is differentiable with respect to $\lambda$ in the strong sense, and

$$
i d \Psi\left(\sigma_{\lambda}\right) / d \lambda=\lim _{N \rightarrow \infty} H(\lambda ; \varrho) U_{N}\left(\sigma_{\lambda}, \sigma_{\lambda_{0}}\right) \Psi .
$$

This implies that $\Psi\left(\sigma_{\lambda}\right)$ belongs to the domain of $\tilde{H}(\lambda ; \varrho)$ and satisfies the Equation (3.7). By the same estimate as (3.9), we can show that $U_{N}^{*}\left(\sigma_{\lambda}, \sigma_{\lambda_{0}}\right)$ is strongly convergent and hence $U\left(\sigma_{\lambda}, \sigma_{\lambda_{0}}\right)$ is unitary.

Remark. The Equation (3.8) can be rewritten in the familiar form

$$
\begin{aligned}
U\left(\sigma_{\lambda}, \sigma_{\lambda_{0}}\right) \Psi= & \sum_{n=0}^{\infty}(-i)^{n}(n !)^{-1} \int_{\sigma_{\lambda_{0}}}^{\sigma_{\lambda}} \ldots \int_{\sigma_{\lambda_{0}}}^{\sigma_{\lambda}} d x_{n} d x_{n-1} \ldots d x_{1} \\
& T_{P}\left[H\left(x_{n} ; \varrho\right) H\left(x_{n-1} ; \varrho\right) \ldots H\left(x_{1} ; \varrho\right)\right]
\end{aligned}
$$

for any $\Psi \in \bigcup \mathfrak{H}_{N}$, where $T_{P}$ is Wick's chronological operator in the sense that it rearranges a product of operators in the same order as of the covering $\left\{\sigma_{\lambda}\right\}$. It has usual expansion in terms of Feynman diagram where two point $\tau$-function is in terms of $T_{P}$-product and has a regulator $\varrho$. The difference between $T_{P}$-product and the usual time-ordered product (based on constant $t$ hyperplanes) tends to 0 as the regulator $\varrho$ approaches to a delta function at the origin. This shows also that the dependence of $U\left(\sigma_{\lambda}, \sigma_{\lambda_{0}}\right)$ on the covering $\left\{\sigma_{\lambda}\right\}$ vanishes in the same limit at least for each term of the expansion (3.8).

\section{§4. Case of Quantum Electrodynamics}

We shall be interested in the regularized Hamiltonian density

$$
H(x ; \varrho)=i e N\left[\tilde{\psi}\left(x ; \varrho_{D}\right) \gamma_{\mu} \psi\left(x ; \varrho_{D}\right)\right] A^{\mu}\left(x ; \varrho_{A}\right) .
$$

The fermion fields generate CAR algebra, which causes no problem. We shall introduce a topology on the ${ }^{*}$-algebra $\mathfrak{U}_{A}$ generated by electromagnetic field $A^{\mu}\left(x ; \varrho_{A}\right)$. (It is a free *-algebra divided by ideals generated by free equation of motion and commutation relations.)

Let $\mathscr{L}=\{F\}$ be the set of all Lorentz frames with a fixed origin. For each $F \in \mathscr{L}$, we consider the Fock space $\mathfrak{H}_{A}(F)$ (with positive metric) where $A_{0}^{-}$and $A_{i}^{+}(i=1,2,3)$ annihilate vacuum $\Psi_{0}(F)$. We shall denote the representative of $Q \in \mathfrak{A}_{A}$ on this space by $Q(F)$. Let $\mathfrak{H}_{A, N}(F)$ be the subspace of $\mathfrak{H}_{A}(F)$ spanned by 
vectors with not more than $N$ particles. We denote by $\tau_{s}(F)$ the topology of $\mathfrak{A}_{A}$ given by the collection of semi-norms $\|Q(F) \Psi\|, \Psi \in \bigcup_{N} \mathfrak{G}_{A, N}(F)$. Let

$$
\tau_{s}=\sup \left\{\tau_{s}(F) ; F \in \mathscr{L}\right\} ;
$$

namely, the weakest topology in the class of topologies each of which is stronger than every $\tau_{s}(F)$. Then $\tau_{s}$ is a Lorentz-invariant topology in $\mathfrak{A}_{A}$. Any vector $\Psi(F) \in \bigcup \mathfrak{S}_{A, N}(F)$ gives a linear functional

$$
f(Q)=(\Psi(F), Q(F) \Psi(F))
$$

which is continuous with respect to $\tau_{s}$. Any vector with a finite number of photons in the Gupta-Bleuler formalism also defines a $\tau_{s}$-continuous linear/iunctional.

We now go over to the algebra

$$
\mathfrak{A}=\mathfrak{U}_{A} \otimes \mathfrak{U}_{D}
$$

of field operators for the whole system. For each $F \in \mathscr{L}$, it has a representation on the Hilbert space

$$
\mathfrak{H}(F)=\mathfrak{H}_{A}(F) \otimes \mathfrak{H}_{D} .
$$

Let $P_{N}(F)$ be the projection onto $\mathfrak{H}_{A, N}(F) \otimes \mathfrak{H}_{D}$ and let

$$
H_{N}(x ; \varrho, F)=P_{N}(F) H(x ; \varrho) P_{N}(F) .
$$

Then the integral

$$
H_{N}(\lambda ; \varrho, F)=\int H_{N}(x ; \varrho, F)\left(\partial x^{0} / \partial \lambda\right) d x
$$

and

$$
\begin{aligned}
U_{N}\left(\sigma_{\lambda}, \sigma_{\lambda_{0}} ; F\right)= & \sum_{n=0}^{\infty}(-i)^{n} \int_{\lambda_{0}}^{\lambda} d \lambda_{n} \int_{\lambda_{0}}^{\lambda_{n}} d \lambda_{n-1} \ldots \int_{\lambda_{0}}^{\lambda_{2}} d \lambda_{1} \\
& {\left[H_{N}\left(\lambda_{n} ; \varrho, F\right) H_{N}\left(\lambda_{n-1} ; \varrho, F\right) \ldots H_{N}\left(\lambda_{1} ; \varrho, F\right)\right] }
\end{aligned}
$$

can be defined and the latter gives the solution of

$$
i d U_{N}\left(\sigma_{\lambda}, \sigma_{\lambda_{0}} ; F\right) / d \lambda=H_{N}(\lambda ; \varrho, F) U_{N}\left(\sigma_{\lambda}, \sigma_{\lambda_{0}} ; F\right) .
$$

In the same way, $H(\lambda ; \varrho)$ and $U\left(\sigma_{\lambda}, \sigma_{\lambda_{0}}\right)$ can be defined by

$$
H(\lambda ; \varrho)=\int H(x ; \varrho)\left(\partial x^{0} / \partial \lambda\right) d x
$$

and

$$
\begin{aligned}
U\left(\sigma_{\lambda}, \sigma_{\lambda_{0}}\right)= & \sum_{n=0}^{\infty}(-i)^{n} \int_{\lambda_{0}}^{\lambda} d \lambda_{n} \int_{\lambda_{0}}^{\lambda_{n}} d \lambda_{n-1} \ldots \int_{\lambda_{0}}^{\lambda_{2}} d \lambda_{1} \\
& {\left[H\left(\lambda_{n} ; \varrho\right) H\left(\lambda_{n-1} ; \varrho\right) \ldots H\left(\lambda_{1} ; \varrho\right)\right] . }
\end{aligned}
$$

This operator $U\left(\sigma_{\lambda}, \sigma_{\lambda_{0}}\right)$ is the solution of

$$
i d U\left(\sigma_{\lambda}, \sigma_{\lambda_{0}}\right) / d \lambda=H(\lambda ; \varrho) U\left(\sigma_{\lambda}, \sigma_{\lambda_{0}}\right),
$$


which is a modification of the Equation (1.1'). The operators $U_{N}\left(\sigma_{\lambda}, \sigma_{\lambda_{0}} ; F\right)$ and $U\left(\sigma_{\lambda}, \sigma_{\lambda_{0}}\right)$ are unitary in the sense that $U^{*}=U^{-1}$, and

$$
U\left(\sigma_{\lambda}, \sigma_{\lambda_{0}}\right)=\lim _{N \rightarrow \infty} U_{N}\left(\sigma_{\lambda}, \sigma_{\lambda_{0}} ; F\right) .
$$

The operators $U\left(\sigma_{\lambda}, \sigma_{\lambda_{0}}\right)$ do not belong to $\mathfrak{A}$. Rather than adding them to $\mathfrak{A}$, we consider the mapping from $\Psi(F) \in \bigcup_{N} \mathfrak{S}_{A, N}(F) \otimes \mathfrak{S}_{D}$ to $\Psi\left(\sigma_{\lambda}\right)=U\left(\sigma_{\lambda}, \sigma_{\lambda_{0}}\right) \Psi(F)$, which induces a linear mapping of continuous linear functionals because $Q_{n} \rightarrow 0$, $Q_{n} \in \mathfrak{A}$, with respect to $\tau_{s}$ implies

$$
Q_{n}(F) U\left(\sigma_{\lambda}, \sigma_{\lambda_{0}}\right) \Psi(F) \rightarrow 0
$$

in $\mathfrak{S}(F)$.

Acknowledgement. The author wishes to thank Prof. H. Araki for his helpful advice on this work.

\section{References}

1. Yosida, K.: Functional Analysis. Berlin-Heidelberg-New York: Springer 1965

2. Kato, T.: J. Math. Soc. Japan 5, $208-234$ (1953); Comm. Pure Appl. Math. 9, 479_486 (1956)

Communicated by H. Araki

Received March 1, 1976; in revised form May 8, 1976 